Category: Teaching \& Learning at times of uncertainty

\title{
Paediatric palliative care: needs assessment for undergraduate medical and nursing education
}

\author{
Wong, K.Y., Victor Li, W.T.V., Yiu, P.Y. \\ The University of Hong Kong
}

\section{Background:}

Paediatric palliative care (PPC) is in its nascent stage despite being an essential component of paediatrics. With its growing importance in healthcare of the $21^{\text {st }}$ century, the expertise, emotional coping and communication skills of healthcare professionals have been greatly challenged. This situation is partially attributed to shortfalls in undergraduate education. This cross-sectional explorative study reveals the PPC-related knowledge, attitude and educational needs of undergraduate preclinical medical and nursing students in Hong Kong.

\section{Method:}

A pretested expert-reviewed survey containing 44 items from 11 categories was conducted to determine respondents' understanding of and perceptions towards PPC. Medical and nursing students without extensive clinical experience were recruited by convenience sampling at the University of Hong Kong. The chi-square test, Fisher's exact test, and descriptive statistics were used to analyse the data.

\section{Results:}

Although only $38 \%$ of the 241 students who completed the questionnaires had heard about PPC, more than two-thirds supported the local development of PPC. They believed that spending time to care for dying children is worthwhile and self-rewarding. However, they generally misunderstood the associated fundamental principles of palliative care and related pain assessments. There were significantly more medical than nursing participants recognised PPC's multidisciplinary nature, but they felt less mentally prepared to discuss death and dying. Overall, $86 \%$ of respondents from both programmes advocated the inclusion of PPC in their curricula.

\section{Conclusions:}

The findings suggested that a comprehensive PPC education including a range of topics is required and welcomed among students. Both curricula should include fundamental PPC palliative concepts and emotional aspects of young patients and their families. Emotional coping and communication skills should be emphasised in medicine, while pain management and multidisciplinary approaches should be taught in nursing. Since this knowledge benefits not only PPC but also the overall healthcare, we strongly encourage to integrate PPC education into both curricula.

Keywords: Palliative care education 